$$
-
$$


THE KLUWER INTERNATIONAL SERIES

IN ENGINEERING AND COMPUTER SCIENCE

ROBOTICS AND VISION

Consulting Editor

Takeo Kanade 


\title{
SHADOWS AND SILHOUETTES IN COMPUTER VISION
}

\author{
by \\ STEVEN A. SHAFER
}

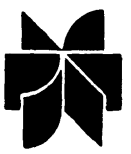

SPRINGER SCIENCE+BUSINESS MEDIA, LLC 
This research was sponsored by the Defense Advanced Research Projects Agency (DOD), ARPA Order No. 3597, monitored by the Air Force Avionics Laboratory under Contract F33615-81-K-1539.

The views and conclusions contained in this document are those of the author and should not be interpreted as representing the official policies, either expressed or implied, of the Defense Advanced Research Projects Agency or the US Government.

\section{Library of Congress Cataloging in Publication Data}

\section{Shafer, Steven A.}

Shadows and silhouettes in computer vision.

(The Kluwer international series in engineering and computer science. Robotics and vision)

Bibliography: $p$.

Includes index.

1. Shades and shadows. 2. Geometry, Descriptive.

3. Cylinders. I. Title. II. Series
QA519.S43 1985
516.2
$85-212$

ISBN 978-1-4419-5197-7

ISBN 978-1-4757-1845-4 (eBook)

DOI 10.1007/978-1-4757-1845-4

Copyright (C) 1985 by Springer Science+Business Media New York

Originally published by Kluwer Academic Publishers in 1985

No part of this book may be produced in any form by print, photoprint, microfilm, or any other means without written permission of the publisher. 
To the memory of Dr. Joseph Shafer, my grandfather. 


\section{Contents}

1. Introduction 1

1.1. The Shadow Geometry Problem 2

1.2. The Gradient Space 4

1.3. Generalized Cylinders 5

1.4. Outline of the Book 7

2. Imaging Geometry and the Gradient Space $\quad 11$

2.1. Imaging Geometry and Terminology 11

2.2. Gradient Space and Orthography 13

2.3. Gradient Space Under Perspective 20

2.4. Conclusions 28

3. The Basic Shadow Problem 29

3.1. Shadow Edge Pairs and the Basic Shadow Problem 29

3.2. Solution of the Basic Shadow Problem $\quad 30$

3.3. Relationships Among the Parameters Supplied in Advance 37

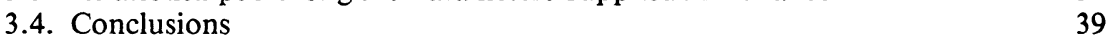

4. Variations in Lighting $\quad 41$

4.1. Light Source in Front of the Camera 42

4.2. Light Source Behind and Below Camera 43

4.3. Light Source Not Infinitely Distant 44

4.4. Position of the Light Source Under Perspective 46

4.5. Line Labels and the Light Source Position 47

4.6. Changing the Number of Light Sources 48

4.7. Conclusions 49

5. Shadows Falling on Polyhedra 51

5.1. Shadows Falling on Polyhedra With No Shadow Edge Discontinuities 51

5.2. Breaks in the Shadow Edge 53

5.3. Constraints in the General Case 55

5.4. Conclusions 56

6. Shadows Cast by Polyhedra

6.1. Polyhedra With Two Visible Surfaces $\quad 57$

6.2. Adding a Self-Shadowed Surface $\quad 59$

$\begin{array}{ll}\text { 6.3. Adding an Illuminated Surface } & 61\end{array}$

6.4. The General Solution for Polyhedral Shadow Geometry 63

$\begin{array}{ll}\text { 6.5. Conclusions } & 65\end{array}$

$\begin{array}{ll}\text { 7. Shadow Geometry for Curved Surfaces } & 67\end{array}$

$\begin{array}{ll}\text { 7.1. Gradients of Curves and Surfaces } & 68\end{array}$

$\begin{array}{ll}\text { 7.2. Shadow Falling on a Curved Surface } & 73\end{array}$

$\begin{array}{ll}\text { 7.3. Shadow Cast by a Curved Surface } & 75\end{array}$

7.4. Conclusions 
$\begin{array}{lr}\text { 8. Generalized Cylinders } & 83\end{array}$

8.1. A Taxonomy of Generalized Cylinders

8.2. Straight Homogeneous Generalized Cylinders 91

8.3. The Equivalence Problem for Shape Descriptions 95

8.4. Surface Normals of an SHGC 99

$\begin{array}{lr}\text { 8.5. Conclusions } & 103\end{array}$

9. Contour Analysis for SHGCs $\quad 105$

9.1. Contours of Tangency for SHGCs 106

$\begin{array}{ll}\text { 9.2. Images of Right SHGCs } & 110\end{array}$

9.3. Contour and Silhouette Analysis for Right Circular SHGCs 112

9.4. Contour and Silhouette Analysis for Right Circular SHGCs (cont.) 120

9.5. Contour and Silhouette Analysis for Other Right SHGCs 126

$\begin{array}{ll}\text { 9.6. Conclusions } & 131\end{array}$

10. Shadow Geometry for Solids of Revolution 133

10.1. Analysis of the Position of the Terminator 134

$\begin{array}{ll}\text { 10.2. Analysis of Surface Normals } & 137\end{array}$

10.3. Aligning the Image for Analysis of Surface Normals 140

10.4. Analyzing the Surface Normals Along the Terminator 141

10.5. Conclusions 145

11. Shadow Geometry and Other Techniques 147

11.1. Shadow Geometry and Other Gradient Space Techniques 147

$\begin{array}{lr}\text { 11.2. Curved Surface Reconstruction } & 149\end{array}$

11.3. Analysis of Range Finder Images of RCSHGCs 151

$\begin{array}{ll}\text { 11.4. Conclusions } & 156\end{array}$

12. Conclusions 157

12.1. Related Problems and Further Work 160

$\begin{array}{ll}\text { Bibliography } & \mathbf{1 6 3}\end{array}$

$\begin{array}{ll}\text { Appendix A. Terminology and Notation } & 169\end{array}$

Appendix B. Basic Shadow Problem in Orthography 171

Appendix C. Basic Shadow Problem in Perspective 175

Appendix D. Catalog of Straight Homogeneous GCs 179

$\begin{array}{ll}\text { Appendix E. The Slant Theorem } & 187\end{array}$

Appendix F. The Pivot Theorem 191

$\begin{array}{ll}\text { Author Index } & 195\end{array}$

$\begin{array}{ll}\text { Subject Index } & 197\end{array}$ 


\section{List of Figures}

Figu re 1.1: Shadows Provide Information for 3-D Shape Recovery 1

Figure 1.2: Concept of a Generalized Cylinder 5

Figure 1.3: Examples of Generalized Cylinders 5

Figure 1-4: Imaging and Shadow Volumes are Generalized Cylinders 7

Figure 2-1: Coordinate System $\quad 12$

Figure 2.2: Gradient of Surface and Surface Normal 15

Figu re 2-3: Gradient of a Vector Under Orthography 15

Figure 2.4: Vector Contained on Two Surfaces 16

Figure 2-5: Two Vectors Contained on a Surface $\quad 17$

Figure 2.6: Gradients of Perpendicular Vectors 18

Figure 2-7: Rotating the Image and the Gradient Space 19

Figure 2-8: Vanishing Point of a Line $\quad 20$

Figure 2-9: Vanishing Line and Gradient of a Surface 22

Figure 2-10: Back-Projection of a Point Onto a Surface 24

Figure 2-11: Vector Contained on a Surface Under Perspective 25

Figure 2-12: Restrictions on the Slope of $L \quad 26$

Figure 2.13: Vector Contained on Two Surfaces Under Perspective 27

Figure 3.1: The Basic Shadow Problem 30

Figure 3-2: Basic Shadow Problem .- Correspondences Labeled 31

Figure 3-3: Basic Shadow Problem .- Illumination Surface 1

Figure 3-4: Gradient Space Constraints from Illumination Surface 1

Figure 3-5: Basic Shadow Problem .- Illumination Surface 2

Figure 3-6: Gradient Space Constraints From Illumination Surface 2

Figure 3-7: Basic Shadow Problem .. Illumination Vector 33

Figure 3-8: Gradient Space Constraints From Illumination Vector $\quad 34$

Figure 3-9: Solution to Basic Shadow Problem 36

Figure 3.10: Basic Shadow Problem .. Edge $E_{\mathrm{OS}}$ Provided 37

Figure 3-11: Redundancy of $E_{\mathrm{OS}}$ With $E_{\mathrm{O} 2}$ and $E_{\mathrm{S} 2} \quad 38$

Figure 3.12: Occurence of the Basic Shadow Problem 39

Figure 4-1: Geometry With Light Source In Front of Camera, Infinitely Far 42 Away

Figure 4-2: Gradient Space Constraints with Light Source in Front of 43 Camera

Figure 4-3: Light Source Behind and Below Camera, Infinitely Far Away 44

Figure 4-4: Point Light Source at Finite Distance 45

Figu re 4-5: Line Labels and Light Source Positions 48

Figu re 4-6: Basic Shadow Problem With Multiple Light Sources 49

Figure 4-7: Two Surfaces With No Light Source 50

Figure 5-1: Shadow Falling On Two Surfaces 52 
Figu re 5.2: Gradient Space Constraints from Two Shaded Surfaces 53

Figu re 5-3: Shadow Falling On Many Surfaces $\quad 54$

Figure 5-4: Shadow Edge With Discontinuites $\quad 54$

Figu re 6-1: Shadow Cast By Simple Polyhedron 58

Figure 6-2: Light Source In a Different Position $\quad 59$

Figure 6-3: Polyhedron With Two Self-Shadowed Surfaces $\quad 60$

Figure 6.4: Polyhedron With Two Illuminated Surfaces 61

Figure 6.5: Additional Parameter Needed for Hidden Shadow-Making 62 Surface

Figure 6.6: Additional Parameter Needed for Non-Trihedral Vertex 63

Figure 6-7: Polyhedron With Additional Invisible Surfaces $\quad 64$

Figure 7.1: Gradient of a Curve and a Tangent Vector 68

Figure 7-2: Gradient of a Curve Under Orthography 68

Figure 7-3: Curve Contained on a Curved Surface $\quad 70$

Figure 7-4: Curve Contained on Two Curved Surfaces $\quad 71$

Figure 7-5: Two Curves Contained on a Curved Surface $\quad 72$

Figure 7-6: Shadow Cast On a Curved Surface $\quad 74$

Figu re 7-7: Gradient Space Constraint Between $G_{X}$ and $G_{11}$

Figu re 7-8: Gradient Space Constraints On Tangent Plaries To $S_{S} \quad 75$

Figu re 7-9: Shadow Cast By a Curved Surface 76

Figure 7-10: Terminators are Unrelated To Surface Orientation 77

Figure 7-11: Gradient Space Constraints on $G_{x} \quad 78$

Figure 7-12: Using $E_{10}$ to Calculate the Gradient of $S_{S} \quad 79$

Figu re 7.13: Gradient Space Constraints From $\boldsymbol{P}_{\mathrm{OX}}$ For Computing $\boldsymbol{G}_{\mathrm{S}} \quad 80$

Figu re 8-1: Taxonomy of Generalized Cylinders 86

Figure 8.2: Characterizing the Ends of a Generalized Cylinder 88

Figure 8-3: Straight Homogeneous Generalized Cylinder 91

Figure 8-4: Linear SHGC 93

Figure 8.5: Circular SHGC 93

Figure 8-6: Polygonal SHGC 93

Figure 8-7: Coordinate Axes for SHGCs $\quad 94$

Figure 8-8: Coordinates of a Point on an SHGC 95

Figu re 8-9: The Equivalent Right SHGC Problem 97

Figure 8-10: The Slant Theorem $\quad 97$

Figure 8-11: The Alternate Axis Problem 98

Figure 8-12: The Pivot Theorem $\quad 99$

Figure 8-13: Surface Normal of an SHGC 100

Figure 8-14: The Corresponding Normal Theorem 101

Figure 8-15: Surface Normals of the Shadow Volume 102

Figure 9-1: Contours and Contour Generators 106

Figu re 9-2: Viewing Direction and Angle $\quad 107$

Figure 9.3: End, Side, and Oblique Views 108

Figu re 9.4: Object and World Coordinate Systems $\quad 110$

Figure 9-5: Image of a Point on a Right SHGC 111

Figure 9-6: Imaging Under Perspective Projection 111

Figure 9-7: Contour Generator on a Right Circular SHGC 113 
Figu re 9-8: Contour Generator in Side View 115

Figu re 9-9: Contour Generator in Near-Side View 116

Figu re 9-10: Contour Generator Singular When Tangent to Line of Sight 117

Figu re 9-11: Contour Generator Split at Near-End View 118

Figu re 9-12: Contour Pieces Correspond to Disjoint Intervals of $s \quad 118$

Figu re 9-13: Aligned Image of an SHGC 120

Figu re 9-14: Contour Analysis Results in Shape Description 121

Figu re 9-15: Contours of a Solid of Revolution are Ambiguous 122

Figu re 9-16: RCSHGC With Near End Flat 125

Figu re 9-17: Tangents at Corresponding Points Intersect on the Axis 126

Figu re 9-18: Contour Generators Need Not Be At Vertical Extrema 128

Figu re 9-19: Crease Contours for a Right Polygonal SHGC 128

Figu re 9-20: Tangents of Crease Contours Intersect on the Axis 129

Figu re 10-1: Contour Generators From Two Points of View 134

Figu re 10-2: Terminator is a Contour Generator for the Light Source 134

Figu re 10-3: Two Angles Define the Illumination Direction 135

Figu re 10-4: Terminator on a Right Circular SHGC 136

Figu re 10-5: Sources of Knowledge About Surface Normals 137

Figu re 10-6: Knowledge of a Single Surface Normal 138

Figu re 10-7: Analyzing a Single Surface Normal 139

Figu re 10-8: Complete and Partial Alignment of the Image 140

Figu re 10-9: Cross-Section of a Right Circular SHGC Through a Point 142

Figure 10-10: Shadow Geometry Provides Surface Normals Along 143 Terminator

Figu re 10-11: Corresponding Point on Tangency Contour 144

Figu re 10-12: At Most One Corresponding Point Exists 145

Figu re 11-1: Shape From Shading 148

Figu re 11-2: Shadow Geometry and Shape From Shading 148

Figure 11-3: Shadow Geometry and Skewed Symmetry 149

Figu re 11.4: Shadow Geometry and Curved Surface Shape Recovery 150

Figu re 11-5: Range Data Yields an Image of Surface Normals 151

Figu re 11-6: Two Surface Normals on the Same Cross-Section 152

Figu re 11-7: Two Surface Normals on Different Cross-Sections 152

Figu re 11-8: Only Compare Surface Normals with Similar Values of $x \quad 153$

Figu re 11-9: Fitting Ellipses to Interpolated Surface Normals 155

$\begin{array}{ll}\text { Figure B-1: The Basic Shadow Problem } & 171\end{array}$

Figure E-1: The Slant Theorem 187

Figure F-1: The Pivot Theorem 191

Figure F-2: Axes in the Proof of the Pivot Theorem 192 


\section{Definitions and Theorems}

Definition 2-1: Orthographic Projection 12

Definition 2-2: Perspective Projection $\quad 12$

Definition 2-3: Surface Gradient 13

Theorem 2-4: Gradient of a Plane 13

Definition 2.5: Vector Gradient 14

Theorem 2-6: Vector Contained on a Surface 14

Theorem 2-7: Vector Contained on Two Surfaces 16

Theorem 2-8: Two Vectors Contained on a Surface 16

Theorem 2-9: Gradients of Perpendicular Vectors and Planes 17

Theorem 2-10: Rotation of the Image and Gradient Space 18

Definition 2-11: Vanishing Point of a Line 20

Definition 2-12: Vanishing Line of a Surface $\quad 21$

Definition 2-13: Vanishing Gradient of an Image Line 23

Theorem 2-14: Point Contained on a Surface 23

Theorem 2-15: Vector Contained on a Surface 24

Theorem 2-16: Vector Contained on Two Surfaces 26

Definition 3-1: Shadow Vector and Edge Pairs 29

Definition 3-2: Basic Shadow Problem 30

Theorem 3-3: Solution of the Basic Shadow Problem 35

Theorem 3-4: Redundancy of Edges in the Basic Shadow Problem 37

Definition 7.1: Gradient of an Curve 68

Theorem 7-2: Curve Contained on a Surface $\quad 69$

Theorem 7-3: Curve Contained on Two Surfaces $\quad 71$

Theorem 7-4: Two Curves Contained on a Surface $\quad 72$

Theorem 8-1: Slant Theorem $\quad 97$

Theorem 8-2: Pivot Theorem 98

Theorem 8-3: Corresponding Normal Theorem 101

Definition 9.1: Contours and Contour Generators 106

Theorem 9-2: Planarity of Contour Generators 108 


\section{Acknowledgments}

The work described herein, and the development of my scientific philosophy, have been inspired and guided by my advisor, Takeo Kanade. His insight, values, and systematic approach to science have contributed to this research directly through his comments and indirectly through the impact he has had on my growth and maturation as a scientist. It has been my great fortune to have written this material under his direction, and my blessing to have been his student throughout my tenure at this university.

I have also been deeply influenced by my other advisors, formal and informal, John Kender, Hans-Hellmut Nagel, D. Raj Reddy, and Bob Sproull. All of these have imparted to me a concern for fundamental and durable contributions to the science of image understanding, an attitude which led to the achievement of the results described in this book.

Several observations described herein are directly attributable to Marty Herman, Bruce Lucas, and David Smith. They and the other members of the Image Understanding project at CMU have provided the remarkably rich and supportive research environment which has made this work both possible and pleasurable. Key observations were also contributed by Tom Binford of Stanford University and Chris Brown of the University of Rochester.

Thanks are due to the system staff in the Computer Science Department at CMU and to Unilogic, Inc., for providing the hardware and software used to pursue and publish this research; to Kitty Fischer for producing the figures for publication; and to Earl Mounts and the librarians in the CMU Engineering and Science Library for assistance in. finding and procuring research materials. Carl Harris at Kluwer Publishers was helpful in preparing the material as a book.

Finally, credit for this work is due in part to Jay Enoch, who brought me to realize that scientific research was my most important professional goal. 
SHADOWS AND SILHOUETTES IN COMPUTER VISION 